\title{
Location-Aware Adaptive Physical Layer Design for Vehicular Visible Light Communication
}

\author{
Gokhan Gurbilek $^{1,2}$, Mertkan Koca ${ }^{1,2}$, Ali Uyrus ${ }^{1}$, Burak Soner ${ }^{1,2}$, Ertugrul Basar ${ }^{1}$ and Sinem Coleri ${ }^{1}$ \\ ${ }^{1}$ Department of Electrical and Electronics Engineering, Koc University, Sariyer, Istanbul, Turkey, 34450 \\ ${ }^{2}$ Koc University Ford Otosan Automotive Technologies Laboratory (KUFOTAL), Sariyer, Istanbul, Turkey, 34450 \\ E-mail: [ggurbilek13, mkoca14, auyrus18, bsoner16, ebasar, scoleri] @ ku.edu.tr
}

\begin{abstract}
Vehicular visible light communication (V2LC) is expected to complement radio frequency (RF) technologies for higher reliability in vehicular connectivity. Since high mobility makes the line-of-sight V2LC channel very dynamic, an adaptive physical layer (PHY) design is required for realizing a rateoptimal and reliable V2LC system. Existing studies on adaptive PHY designs have mostly considered indoor scenarios with low mobility and require a feedback channel for both reporting the received signal-to-noise ratio (SNR) to the transmitter and channel equalization (CE), which increases system complexity and introduces overhead. This paper presents a novel lowcomplexity adaptive PHY design that provides rate-optimal and reliable V2LC without a feedback channel. The proposed design utilizes a priori measurements of the BER with respect to SNR, which are static for V2LC on the road. SNR is predicted in real-time based on the relative locations of the transmitting (TX) and receiving $(\mathrm{RX})$ vehicles using a path loss model based on a priori measurements of the SNR-distance relationship and the polar beam pattern for a given $\mathbf{T X} / \mathbf{R X}$ pair, in a given setting. The proposed design is validated via night-time experiments with On-Off-Keying (OOK), 4-Pulse-Position Modulation (4-PPM) and Direct Current-Biased Optical OFDM (DCO-OFDM). The proposed location-aware adaptive PHY design can be expanded for general reliable rate-optimal V2LC use by updating the path loss model with additional measurements for different settings.

Index Terms - Vehicular communication, visible light communication, vehicle to vehicle communication, Adaptive visible light communication (VLC), Location-aware VLC
\end{abstract}

\section{INTRODUCTION}

Vehicular visible light communication (V2LC) is expected to complement radio frequency (RF)-based vehicular communication technologies such as IEEE $802.11 \mathrm{p} /$ Dedicated Short Range Communication (DSRC) and Cellular Vehicular to Everything Communication (C-V2X) for higher reliability [1], [2]. V2LC utilizes the readily available light emitting diode (LED) head/tail/fog lights and thus, with very little extra cost, acts as a high-rate and reliable alternative in crowded vehicular environments where RF communications suffer.

Channel parameters change dynamically in a highly mobile V2LC channel. Therefore, an adaptive physical layer (PHY) design is necessary for rate-optimal and reliable V2LC. For reliable communication, the adaptive PHY requires a target biterror-rate (BER) below the Forward Error Correction (FEC) limit of $3 \times 10^{-3}$. In such an adaptive PHY design, the transmitter (TX) needs to know the instantaneous signal-to-noise ratio (SNR) at the receiver (RX) and the BER at that SNR for a given waveform. Given the SNR, the adaptive PHY selects among the available modulation schemes under the target BER to maximize the spectral efficiency.

Existing adaptive VLC PHY designs have employed bit/power loading [3], [4], [5] and bandwidth adjustments [6] utilizing feedback channels for reporting the RX SNR back to the TX. However, these designs are not desirable for a V2LC implementation because of three reasons. First, bit/power loading and bandwidth adjustment algorithms in these existing works have a high degree of complexity, introducing an undesired high computational overhead. Second, since existing algorithms consider low mobility environments where path loss changes slowly, they do not have to update their path loss model via the feedback channel frequently and therefore, the rate loss incurred by the feedback channel is low. This rate loss is very significant in the high-mobility V2LC case and thus eliminating the need for a feedback channel is highly desirable for V2LC. Finally, existing algorithms consider indoor environments where the BER vs. SNR characteristics for waveforms change dynamically primarily due to multi-path effects. Therefore for indoor environments and mobile TX/RX, the use of feedback channel is a must since instantaneous BER vs. SNR measurements are impractical. However, in the V2LC setting, the BER vs. SNR relationships deviate from the ideal Additive White Gaussian Noise (AWGN) versions primarily due to the low-pass channel frequency response (i.e. LED response) and the non-linearity introduced by the TX drivers. Since both of these major effects are static on the road [7], [8], the need for a feedback channel can be removed by introducing a RX SNR prediction algorithm at TX.

In this paper, a novel low-complexity adaptive PHY design for rate-optimal and reliable V2LC without a feedback channel, is presented. The proposed design predicts the RX SNR at the TX side in real-time based on the relative RX location and a path loss model based on simple associated a priori measurements. Then, the design makes a selection among different modulation methods (for which it holds spectral efficiency and BER vs. SNR information in its library tabulated via further simple a priori measurements) to sustain the highest possible rate at that SNR while ensuring reliable communication by keeping BER $<$ FEC limit.

The rest of the paper is organized as follows. Section II provides the system model. The proposed adaptive PHY design, its SNR prediction and modulation method selection algorithms and the methodology for the a priori measure- 


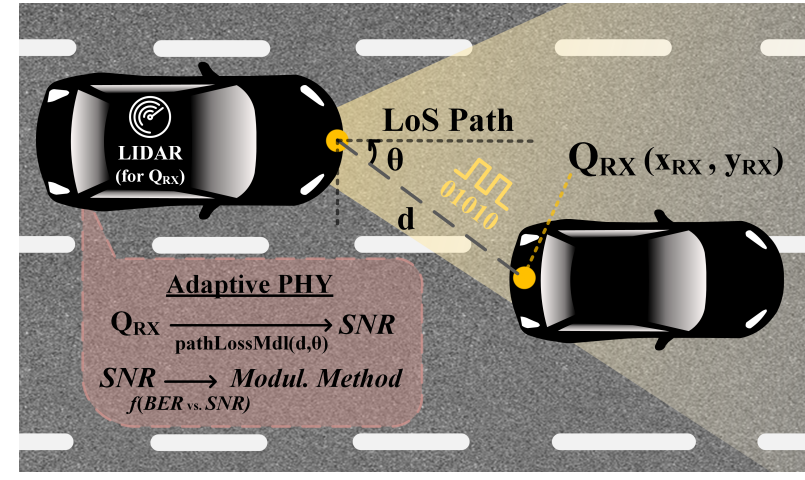

Figure 1: Location-aware adaptive physical layer design and system model for vehicular visible light communications

ments they require are presented in detail in Section III. The proposed design is experimentally validated in Section IV for a given TX/RX pair in a night-time setting with On-Off Keying (OOK), 4-Pulse Position Modulation (4-PPM) and Direct Current-Biased Optical OFDM (DCO-OFDM) methods. Section $\mathrm{V}$ provides the conclusion and future work.

\section{SySTEM MODEL}

The system model considers vehicles that are equipped with V2LC TX and RX hardware: LED head/tail/fog lights and associated drivers for TX and photodiodes with associated optics and amplifiers for RX. TX polar beam pattern is dependent on the LED and its beam-shaping optics. RX polar pattern is assumed to be flat over its field-of-view for simplicity.

VLC is an intensity modulation / direct detection (IM/DD) based technology and it can utilize modulation methods which use positive-definite signals on LED intensity modulation with different BER and spectral efficiency performances. When the reliability condition of $\mathrm{BER}<\mathrm{FEC}$ limit is met, modulation methods with higher spectral efficiency is targeted. Low-pass channel frequency response and the TX LED driver nonlinearities cause inter-symbol interference (ISI), which results in a shift towards more error on the BER vs. SNR curves as shown in [5]. In contrast to the application scenario in [5], these effects are static for the V2LC channel.

The vehicles are additionally assumed to be equipped with sensors such as Radio Detection And Ranging (RADAR) and/or Light Detection And Ranging (LIDAR) which can find the relative location of other vehicles on the road. Using this real-time location information and the path loss model based on a priori SNR-distance and TX polar beam pattern introduced in this work, the adaptive PHY predicts the RX SNR on the TX computer. The system model and a conceptual representation of the proposed adaptive PHY design, which will be presented in detail in Section III, is depicted in Fig. 1.

\section{Proposed AdAptive PHY DESIGN}

This section presents the proposed low-complexity adaptive PHY design which runs at TX. The contributions of the proposed design are; a methodology for predicting the RX SNR at TX based on real-time RX location, and a methodology for choosing the most spectrally efficient modulation method among methods that can attain BER $<$ FEC limit at that SNR. Both features of the proposed design rely on libraries and models built on one-time-only a priori measurements.

\section{A. Real-Time SNR Prediction}

The instantaneous RX SNR prediction at TX removes the need for a feedback channel. A path loss model, which does the prediction based on the current setting (i.e. ambient light and environmental conditions) and the relative location of the $\mathrm{RX}$ is required for the prediction algorithm. The adaptive PHY design then uses the real-time location information supplied by the LIDAR/RADAR and the path loss model to predict the SNR.

The path loss model is built via two a priori measurements for a given TX/RX pair: SNR vs. distance curve for each modulation method and polar beam pattern of the head/tail/fog light. SNR vs. distance curve measurements are needed to be repeated for different settings and interpolated between those discrete measurements in order to have a path loss model that is valid over a wide range of environmental settings [7]. Since the polar beam pattern measurement is inherently a differential measurement, it only needs to be done once and is valid for all settings. Employing both measurements, a map of SNR values with respect to the location of the RX inside the TX field-of-view is obtained for all settings.

The expression for predicting the RX SNR at TX using the above-mentioned terms for a given modulation method is as follows:

$$
\Gamma_{d, \theta, M M(\text { elec })}[d B]=\Gamma_{d, M M(\text { elec })}[d B]-\psi(\theta)[d B]
$$

where $\mathrm{d}$ is the distance between vehicles, $\theta$ is the angle from the boresight, $\Gamma_{d, \theta, M M(\text { elec })}$ and $\Gamma_{d, M M(e l e c)}$ are the predicted electrical SNR value for the given modulation method (MM $\in$ OOK, 4-PPM and DCO-OFDM) as a function of distance and angle, and distance only, respectively. $\psi(\theta)[d B]$ is the angular attenuation in $\mathrm{dB}$ scale.

\section{B. Selecting Reliable and Rate-Optimal Modulation}

After obtaining the SNR value, the proposed design first evaluates the $\mathrm{BER}<\mathrm{FEC}$ limit condition of all modulation methods for that SNR, and then chooses the method with the highest spectral efficiency among them. TX sends the waveform with this chosen modulation method. RX receives the waveform and chooses a demodulation method by the received SNR.

In order to carry out this evaluation, the design needs to know the BER vs. SNR characteristics of each modulation method in its library. The environmental conditions and the intervehicular distance primarily affect the received signal level as relative frequency dependent characteristics of the V2LC channel resembles a typical $-10 \mathrm{~dB} /$ decade after its $3 \mathrm{~dB}$ cutoff [7], [8]. Therefore, a one-time-only a priori measurement of SNR vs. BER curve for each modulation method is enough. Using this library, the proposed design creates a possible list of modulation methods that satisfy the aforementioned reliability constraint, then chooses the most spectrally efficient one. 


\section{IMPLEMENTATION OF PROPOSED DESIGN}

This section initially presents the experimental setup used for the system implementation and practical details about the three measurements: SNR vs. distance curve for each modulation method, polar beam pattern of the headlight, and SNR vs. BER curve for each modulation method. Afterwards, using the introduced path loss model and the BER vs. SNR library built on the measurements, the proposed design is constructed and validated considering reliability and rate-optimality.

The proposed location-aware adaptive design needs SNR vs. distance curve measurements under different settings to be valid for general V2LC use. The relative frequency dependent channel characteristics of V2LC channel remains stable however, the received signal power is affected by the environmental conditions [8]. Hence, validating the proposed design in only one setting is sufficient. Night-time is selected for this paper since the low ambient noise allows for a more clear presentation of the proposed idea. Furthermore, this paper has considered 4-PPM, OOK and $M$ - ary Quadrature Amplitude Modulation (QAM) DCO-OFDM since they represent different extremes in spectral efficiency and BER characteristics among known modulation methods in literature. The spectral efficiency of OOK [9], 4-PPM [9] and $M$-QAM DCO-OFDM [10] provided in the Table I.

\begin{tabular}{ll}
\hline Modulation Type & $\eta$ (bits/s/Hz) \\
\hline 4-PPM & 0.5 \\
\hline 4-QAM DCO-OFDM & 0.969 \\
\hline OOK & 1 \\
\hline 16-QAM DCO-OFDM & 1.938 \\
\hline 64-QAM DCO-OFDM & 2.906 \\
\hline
\end{tabular}

Table I: Spectral efficiency values for modulation methods used for the implementation of the proposed design

\section{A. Setup}

Three experiments using two different setups are required. The first measurement setup is used for both extracting SNR vs. BER curve of each modulation, and extracting the relationship between SNR and the inter-vehicular distance. The second setup is used for measuring the polar beam pattern of the light source, the TX LED.

For the first setup, TX and RX vehicles are equipped with TX and RX V2LC front-ends. The TX front-end consists of a computer, a Universal Software Radio Peripheral (USRP) 2920 equipped with an LFTX daughter-board and an Ettus Global Positioning System disciplined oscillator (GPSDO), a custommade current-mode LED driver, a Rigol DP832 Power Supply and a commercial off-the-shelf (COTS) automotive fog LED light. The transmitter PHY implementation of the modulation methods are prepared on LabVIEW and fed to the USRP. The output of the USRP is delivered to the custom-made LED driver. The operating range of the LED driver is selected such that LED current and LED optical power have a linear relation. Finally, the current flowing through the fog LED light gets converted into optical intensity and meets the channel.

The receiver front-end consists of a Hamamatsu S3884 C5331 avalanche photodiode (APD), a Rigol DP832 Power

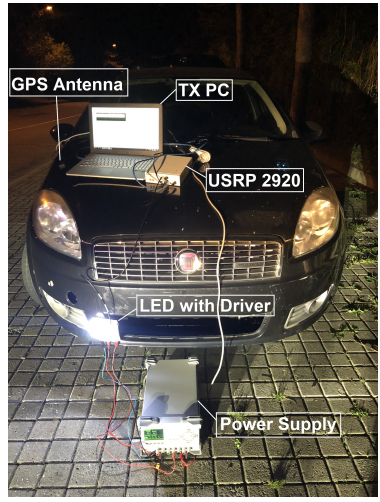

(a)

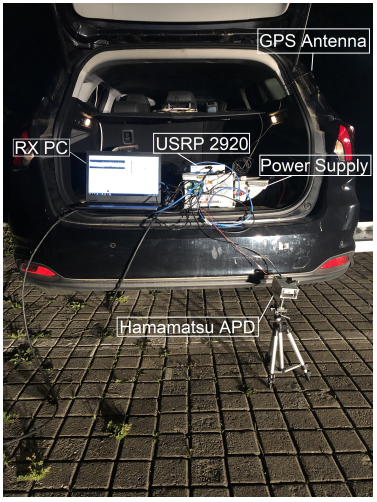

(b)
Figure 2: (a) Transmitter Measurement Setup (b) Receiver Measurement Setup

Supply, a USRP 2920 equipped with an LFRX daughter-board and an Ettus GPSDO, and a computer. The photodetector captures the signal transmitted through the channel and feeds it directly to the USRP, which is connected to the computer via a gigabit ethernet cable. The real-time demodulation of the signal is handled on LabVIEW. The synchronization between the transmitter and the receiver vehicles are achieved via the GPSDOs connected to the USRPs. The two measurements, SNR vs. BER curves and SNR vs. inter-vehicular distance relationship for each modulation method are quantified using the first measurement setup by logging the BER and SNR data for different inter-vehicular distances.

The second setup aims to find the polar beam pattern of the fog LED light. The TX front-end consists of a Rigol DG4202 Arbitrary Waveform Generator (AWG), the custommade LED driver, a Rigol DP832 Power Supply and a COTS automotive LED fog light. A sinusoidal signal produced by AWG at $100 \mathrm{kHz}$ is supplied to the LED driver. The RX front-end consists of a Hamamatsu S3884 - C5331 APD, a Rigol DP832 Power Supply and a Agilent InfiniiVision DSO$\mathrm{X}$ 3034A Oscilloscope. The output of the APD is connected to the oscilloscope. For the measurement, the TX and RX units are separated at $1 \mathrm{~m}$ on their line-of-sight $(\mathrm{LoS})$ path with $0^{\circ}$ angle. Then, the LED is swept from $0^{\circ}$ to $45^{\circ}$ on the perpendicular plane to the direct LoS path of $1 \mathrm{~m}$ distance. At every angle, the received power of the APD output signal is recorded on the oscilloscope. The normalized power of the output signal with respect to varying incident angle is considered as the polar beam pattern of the LED fog light.

\section{B. Results}

The relationship between the inter-vehicular distance and SNR is given in Fig. 3. As seen from this figure, the difference in the constellation size does not affect the inter-vehicular distance vs. SNR relation of $M$-QAM DCO-OFDM waveform. 4-PPM and OOK exhibit different inter-vehicular distance vs. SNR characteristics. The expressions defining the intervehicular distance vs. SNR relation of each modulation method are derived using interpolation. 


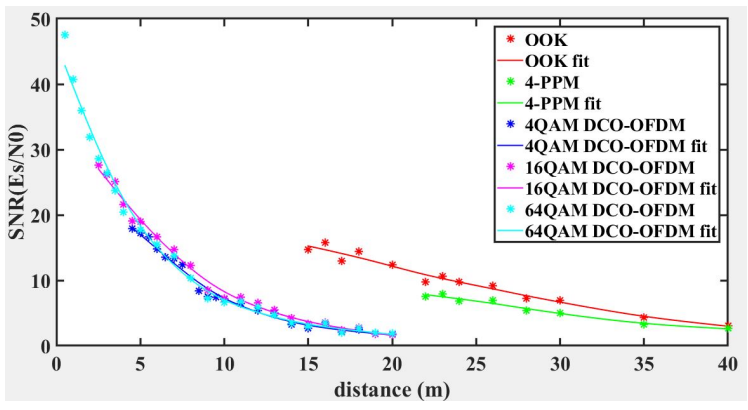

Figure 3: Inter-vehicular distance vs SNR performances of modulation methods

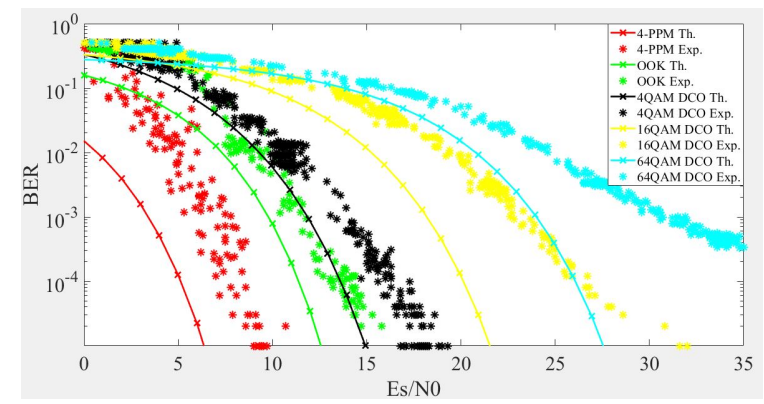

Figure 4: SNR(Es/N0) vs BER performances of modulation methods

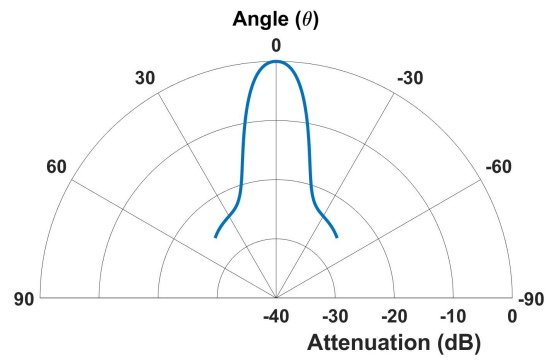

Figure 5: Polar beam pattern of LED fog light

\begin{tabular}{cc}
\hline Modulation Type & SNR Intervals (dB) \\
\hline 4-PPM & $5.8-11.9$ \\
\hline OOK & $11.9-14.1$ \\
\hline 4-QAM DCO-OFDM & $14.1-23$ \\
\hline 16-QAM DCO-OFDM & $23-26.6$ \\
\hline 64-QAM DCO-OFDM & $26.6-\infty$ \\
\hline
\end{tabular}

Table II: Adaptive modulation method selection with respect to $\operatorname{LoS} \mathrm{SNR}(\mathrm{Es} / \mathrm{N} 0)$

The RX signal experiences attenuation proportional to the angular deviation of its position from the TX direct LoS path. The exact amount of that attenuation depends on the polar beam pattern of the TX. Fig. 5 shows the polar beam pattern of TX LED. The angular attenuation of the signal is derived as a function of angle namely $\psi(\theta)[d B]$.

Fig. 4 provides the SNR vs. BER performance of the considered modulation methods. Viewed together with the theoretical AWGN curves, it is evident that the measured curves additionally capture the ISI-induced effects (due to the LED frequency response) which shift the curves to the right, and the non-linearity-based saturation effects (especially visible at the far right for 64-QAM in Fig. 4). For the adaptive design, among methods that can attain $\mathrm{BER}<10^{-3}$, the modulation method with the highest spectral efficiency is selected. A look up table is constructed for both the proposed design in Table II for the given night-time setting.

\section{CONCLUSION AND Future WORK}

V2LC provides a reliable alternative solution in LoS communication in cases of crowded vehicular settings where RF communications suffer. The high mobility of vehicles necessitates an adaptive PHY design for reliable and rateoptimal communication. In this paper, a novel low-complexity, location-aware adaptive PHY design that attains reliable, rateoptimal V2LC without a feedback channel has been proposed. Utilizing only three simple a priori measurements, the proposed design predicts the RX SNR on the TX in real-time and chooses the modulation method that attains reliable communication (i.e. BER $<$ FEC) at that SNR with the highest spectral efficiency. The proposed adaptive PHY design has been validated in night-time conditions for a given TX/RX pair. The proposed design can easily be adapted to any TX/RX pair and expanded for general V2LC use under different settings with additional iterations of the same measurements under those settings. Validation of the methodology for expanding the proposed design to general V2LC use and performance comparison with an adaptive PHY design that utilizes a feedback channel are planned for future work.

\section{ACKNOWLEDGEMENT}

This work was supported by Ford Otosan.

\section{REFERENCES}

[1] W.-H. Shen and H.-M. Tsai, "Testing vehicle-to-vehicle visible light communications in real-world driving scenarios," in Vehicular Networking Conference (VNC), 2017 IEEE, pp. 187-194, IEEE, 2017.

[2] G. Blinowski, "Security of visible light communication systemsa survey," Physical Communication, vol. 34, pp. 246-260, 2019.

[3] O. Gonzalez, R. Perez-Jimenez, S. Rodriguez, J. Rabadan, and A. Ayala, "Ofdm over indoor wireless optical channel," IEE Proceedings Optoelectronics, vol. 152, pp. 199-204, Aug 2005.

[4] L. Wu, Z. Zhang, J. Dang, and H. Liu, "Adaptive modulation schemes for visible light communications," Journal of Lightwave Technology, vol. 33, 012015.

[5] L. Grobe, A. Paraskevopoulos, J. Hilt, D. Schulz, F. Lassak, F. Hartlieb, C. Kottke, V. Jungnickel, and K.-D. Langer, "High-speed visible light communication systems," Communications Magazine, IEEE, vol. 51, pp. 60-66, 122013.

[6] C. Chen, W.-D. Zhong, and D. Wu, "Indoor ofdm visible light communications employing adaptive digital pre-frequency domain equalization," 062016.

[7] G. Gurbilek, M. Koca, B. Turan, and S. C. Ergen, "Poster: Vehicular vlc experimental modulation performance comparison," in 2018 IEEE Vehicular Networking Conference (VNC), pp. 1-2, Dec 2018.

[8] B. Turan, G. Gurbilek, A. Uyrus, and S. C. Ergen, "Vehicular vlc frequency domain channel sounding and characterization," in 2018 IEEE Vehicular Networking Conference (VNC), pp. 1-8, IEEE, 2018.

[9] T. Y. Elganimi, "Performance comparison between ook, ppm and pam modulation schemes for free space optical (fso) communication systems: analytical study," International Journal of Computer Applications, vol. 79, no. 11, 2013.

[10] S. Dimitrov, S. Sinanovic, and H. Haas, "A comparison of ofdm-based modulation schemes for owc with clipping distortion," in 2011 IEEE GLOBECOM Workshops (GC Wkshps), pp. 787-791, IEEE, 2011. 